\title{
LE BUTIN DE GUERRE ET LA TUNIQUE DE NESSUS: TRADUIRE LE RAPPORT AU FRANÇAIS CHEZ MALIKA MOKEDDEM ET ASSIA DJEBAR
}

\author{
Désirée Schyns \\ Université de Gand \\ desiree.schyns@hogent.be
}

\begin{abstract}
Résumé: Cet article développe le point de vue du traducteur qui veut rendre les aspects de multilinguisme dans les textes dits postcoloniaux. En l'occurrence il s'agit de deux auteurs de la diaspora algérienne vivant dans le pays de l'ancien colonisateur. Nous approchons le multilinguisme, ou l'hybridité, à l'aide du concept de l'étranger selon Ricoeur et démontrons que " l'étrangeté " dans deux textes de Mokeddem et Djebar, est liée à un rapport spécifique à la langue française qui découle de la colonisation. Sujet qu'elles thématisent elles-mêmes. La traduction littéraire en néerlandais de quelques fragments pris dans N'zid (2001) de Mokeddem et de L'amour la fantasia (1985) de Djebar, sera évoquée pour mieux comprendre et analyser la forme (métaphores, jeu de mots, choix de mots) que les auteurs ont choisie pour exprimer leur relation à la langue française. Cette relation sera analysée en réfléchissant à la traduction de termes ou d'expressions complexes et polysémiques souvent forgés par les auteurs elles-mêmes.
\end{abstract}

Mots-Clés: Traduction littéraire, traduction de littératures postcoloniales, Djebar, Mokeddem, Diaspora algérienne.

\begin{abstract}
Migratory movements in the world have led to the formation of diasporas and immigrants cultures that transform both societies and immigrants themselves, while contributing to global or transnational identities and cultures. The development of these globalized cultures erodes concepts of culture and identity based on binary oppositions. This article shows the point of view of a translator from French into Dutch confronted with plural identity in postcolonial translation. The textual hybridism in the work of Francophone Algerian writers Assia Djebar and Malika
\end{abstract}


Mokeddem can be contextualized with the help of the concept of Ricoeurs "l'étranger". I want to show that the "strangeness" in style that has to find its way in translation, is linked to a specific relation to French as a result of colonialism. Mokeddem thinks of French as a spoil of war, or a "butin de guerre" as a result of 130 years of French colonisation and the Independence War (1954-1962). Djebar on the other hand considers French as a poisonous gift, as "la tunique de Nessus".

Keywords : Translation Literature, Post-colonial literature translation, Djebar, Mokeddem, Algerian Diaspora.

\section{Introduction}

Malika Mokeddem (Kénadsa, 1949) et Assia Djebar (Cherchell, 1936) ont en commun de pratiquer une écriture qui se caractérise par une forme de multilinguisme. Comme d'autres écrivains francophones, elles présentent une vision migratoire dans leurs textes et transgressent les barrières d'une littérature homogène et nationale. Langue et patrie ne font pas un tout chez elles. De plus leur conception de la relation entre l'arabe dialectal de l'Algérie et le français, leur langue d'écriture, influe sur la littérature qu'elles créent. Non seulement la langue d'écriture transmet un monde culturel de références et "d'étrangeté ", mais il y aussi le monde culturel de la langue maternelle qui sous-tend le français. Selon Dora Sales Salvador ${ }^{1}$ la texture hybride de la littérature postcoloniale contribue à la création d'une vision collective et constructive de l'immigration. Les littératures multiculturelles issues du colonialisme offrent en plus des points de vue intéressants pour l'étude de la traduction grâce à leur hybridité, métissage et multilinguisme². Souvent la traduction se complique dans le cas de textes hybrides qui véhiculent encore plus " l'étranger " que les textes littéraires " non-métissés".

Dans cet article, je vais certes évoquer la traduction littéraire mais surtout comme un moyen de comprendre et analyser la forme 
que Malika Mokeddem et Assia Djebar ont trouvé pour exprimer leur étrangeté par rapport au français. Je m'intéresse à l'imbrication de la culture et de la langue, de style et de contenu donc. J'aimerais démontrer que l'étrangeté dans les textes de Mokeddem et Djebar est liée à un rapport spécifique à la langue française qui découle de la colonisation. Rapport de pouvoir qu'elles thématisent elles-mêmes.

\section{L'étranger selon Ricoeur}

Pour donner un contexte au mot " étranger ", une citation du philosophe français Paul Ricoeur servira de fil rouge. Dans un essai publié en 2004, intitulé "Défi et bonheur de la traduction "3, Ricoeur définit la notion de " l'étranger " dans le contexte d'un texte à traduire :

Deux partenaires sont en effet mis en relation par l'acte de traduire, l'étranger- terme couvrant l'oeuvre, l'auteur, sa langue- et le lecteur destinataire de l'ouvrage traduit. Et, entre les deux, le traducteur qui transmet, fait passer le message entier d'un idiome dans l'autre.

C'est dans cette inconfortable situation de médiateur que réside l'épreuve en question. Franz Rosenzweig a donné à cette épreuve la forme d'un paradoxe. Traduire, dit-il, c'est servir deux maîtres: l'étranger dans son oeuvre, le lecteur dans son désir d'appropriation.

Auteur étranger, lecteur habitant la même langue que le traducteur. Ce paradoxe relève en effet d'une problématique sans pareille, sanctionnée doublement par un voeu de fidélité et un soupçon de trahison. (p. 8-9)

Le terme étranger est un terme qui couvre l'œuvre, l'auteur et sa langue. Ceci vaut donc pour n'importe quel auteur qu'on traduit d'une langue dans une autre, mais l'étrangeté sera dédoublée dans 
le cas d'auteurs francophones comme Mokeddem ou Djebar. Les deux auteurs écrivent en français, mais leur français est coloré et " épicé " d'autres couches culturelles de leur pays d'origine, en l'occurrence l'Algérie. Leur français constitue un tissu riche nourri de différentes cultures, ce qui rend le français à traduire d'une autre façon étrangère que la langue d'un auteur " non-métissé ”.

\title{
Malika Mokeddem et le butin de guerre
}

Dans un entretien avec Christiane Chaulet-Achour et Lalia Kerfa, publié dans Malika Mokeddem : Envers et contre tout ${ }^{4}$, Mokeddem évoque le métissage par le biais du français, une langue qui est devenue sienne du fait de la colonisation. L'auteur aimerait coloniser le français à son tour :

\begin{abstract}
Pour faire rire mes lecteurs, je leur dis souvent : la langue française est venue me coloniser. Maintenant, c'est à mon tour de la coloniser ! Pas pour dire " mes ancêtres, les Gaulois "... comme lorsque j'étais enfant, mais pour y être nomade et, au gré de mes envies lui imprimer la lenteur, la flamboyance des contes de l'oralité, l'incruster de mots arabes dont je ne peux me passer. (p. 29)
\end{abstract}

Le français est pour ainsi dire un butin de guerre, tombé entre les mains des Algériens après le départ des Français en 1962. L'image du butin de guerre vient de l'écrivain francophone algérien Kateb Yacine.

L'étrangeté constitue dans le cas de Mokeddem un rapport à la langue française. Le texte " colonisé " en français est étranger pour un lectorat francophone à cause de la flamboyance déjà mentionnée et de l'incrustation de mots arabes. Comment rendre ces aspects en traduction néerlandaise, étant donné que le néerlandais est une langue qui n'est pas aussi familière avec l'arabe que 
le français ? Je réfère à des mots comme bled ou toubib, mais je ne m'arrêterai pas à ces aspects de la traduction. Je ne m'arrête pas non plus à la " traduction" de références historiques, comme OAS, ou autres références à la guerre d'Algérie, fait historique plus ou moins inconnu aux Pays-Bas et en Flandre.

J'aimerais plutôt explorer quelques extraits de textes qui démontrent l'imbrication de l'étranger forgé dans la langue française. Là où le choix de mots et d'images renvoie à la vision personnelle de l'auteur, à sa façon d'épicer le français et de créer un espace dans la langue française. Cette vision est nourrie par sa culture métissée et par sa volonté de déconstruire des oppositions binaires. Les citations viennent du roman N'zid, mot arabe qui signifie je continue, ou je nais, et que j'ai traduit en néerlandais en 2003 par Un Ulysse féminin, $n^{\prime} z i d{ }^{5}$. Dans ce roman, Mokeddem a voulu trouver une forme littéraire pour exprimer la violence de la guerre civile qui faisait rage dans son pays natal dans les années 90 . Il me semble que Mokeddem, qui vit en exil en France depuis la fin des années 70, essaie de trouver dans ce roman un nouveau pays imaginaire dans la langue française. Le roman est une quête d'identité hybride $^{6}$. Dans le fragment suivant Mokeddem joue avec les sons du français comme s'il s'agissait d'un poème et en même temps elle réfléchit sur le thème de l'étranger : Nora est la narratrice qui se réveille sur son voilier, devenue amnésique à la suite d'un acte de violence perpétré contre elle. Nora a une mère algérienne, un père irlandais et vit en France :

Nora explique qu'il faut d'abord être de quelque part pour se sentir étrangère ailleurs. Dans le mot étrangère il y a une ère en trop. Un espace-temps où s'entend une aire, une terre. (p. 161)

Ce qui est difficile à rendre dans ce fragment de français " colonisé " par Mokeddem c'est le jeu de mots avec " étrangère " et " il y a une ère en trop " et " où s'entend une aire, une terre". 
Quand j'ai traduit ce fragment, j'ai tenu compte du fait qu'il s'agit bel et bien du contenu des mots comme sol, étrangère, terre. Le texte parle de patrie, langue maternelle, nomadisme et est déterminé par la culture métissée de l'auteur. C'est un texte qui résonne de plusieurs langues, de différentes cultures et aussi de différents lieux géographiques comme le désert (lieu de naissance de l'auteur) et de la Méditerranée, l'espace géographique où se déroule la narration. Comme traductrice j'ai dû réécrire le texte ; j'ai identifié le mots le plus important que j'ai voulu absolument garder, comme " terre " et j'ai aussi essayé de jouer un peu avec ce mot en forgeant un autre mot. Avec " taal " et " tong " en néerlandais, j'ai choisi pour "langue de terre" jouant ainsi avec la double résonance de langue en français (ce que l'on parle et l'organe), ce qui donne " tongtaal " en néerlandais pour compenser la perte du jeu de mots en français.

L'espace imaginaire qu'évoque Mokeddem est en effet une lange de terre, l'espace de l'isthme, un espace entre les deux. En exergue du roman on trouve un poème du poète francophone libanais Adonis : "Poète tu n'écris ni le monde ni le moi/ tu écris l'isthme/ entre les deux. "Nora navigue sur la Méditerranée où se trouvent des isthmes comme le Détroit de Gibraltar et le Détroit ou Canal de Corinthe. Cet espace géographique se reflète dans la langue d'écriture de Mokeddem dans N'zid. Dans le fragment suivant l'auteur joue avec la double résonance en arabe du mot du titre, " n'zid" :

"N'zid?": "Je continue?", et aussi: "Je nais." Elle aime la sonorité de ce mot, n'zid. Elle aime l'ambivalence qui l'inscrit entre commencement et poursuite. Elle aime cette dissonance, essence même de son identité. N'zid, elle aime la voix qui la reconnaît de cette langue, elle qui croyait que son physique était de nulle part.

Dans le mot " commencement" on trouve l'écho de " n'zid " (je nais), tout comme dans " poursuite" (je continue). Comme 
Nora (qui pense que son physique était de nulle part) les mots français veulent fuir chaque forme d'ancrage et d'enracinement. Dans la citation on voit que le mot " dissonance " est une partie essentielle de l'identité de Nora. L'ambivalence, la polysémie et la pluralité du mot “ n'zid " se reflètent dans l'identité du personnage principal.

A l'aide d'oxymores l'auteur essaie d'ouvrir la langue à d'autres horizons, comme dans la citation suivante :

L'attachement à une patrie ne symbolise pour elle qu'un état de souffrance : celui de son père, celui de Zana, celui qui l'a privée d'une mère. Il est une menace qu'elle foule aux pieds. Le sol quel qu'il soit ? Entrelacs de lignages que tissent les pas, que balisent des visages, des affections. Elle, elle traverse des juxtapositions d'espaces de langage,_de moments de densité, de tonalités différentes pour se tenir toujours dans la marge. La marge est son lieu privilégié, à la fois refuge et poste d'observation. La marge métamorphose les êtres en vigiles. Nora y apprend les vertiges des ruptures, les blessures de la liberté, l'ampleur salvatrice du doute. (p. 173)

Mokeddem joue avec les sons des mots français et crée maintes allitérations et rimes intérieures qui sont difficiles à rendre dans une langue germanique comme le néerlandais : " sol " et " soit ", " entrelacs de lignages que tissent", " balisent des visages ", " ampleur salvatrice". Elle jette une nouvelle lumière sur des oppositions binaires comme centre et marge, étranger et français de souche, nomadisme et ancrage. Nora se sent bien dans la marge et célèbre le doute au lieu de la certitude. Ne pas appartenir à un seul pays est un avantage au lieu d'un destin malheureux.

Ainsi Mokeddem traverse la langue française, son butin de guerre, pour créer des espaces inattendus et pour déconstruire des visions manichéennes. 


\section{Assia Djebar et la tunique de Nessus}

Contrairement à Mokeddem, Assia Djebar est issue de la bourgeoisie citadine. Son père était instituteur, comme l'écrivain algérien francophone Mouloud Feraoun ou Salah Ould Aoudia, ou le père de l'écrivain algérien francophone Maïssa Bey. Feraoun et Ould Aoudia ont été assassinés par l'OAS pendant la guerre d'Algérie et le père de Maïssa Bey a été torturé à mort par des commandos spéciaux de l'armée française pendant la même guerre de décolonisation.

Comme Mokeddem, Djebar a fréquenté l'école française pendant la colonisation. Et il va sans dire que cette fréquentation a eu une grande influence sur son écriture : sa langue d'écriture est devenue, malgré elle, le français. Par ses grands-parents du côté maternel, Djebar a une culture berbère. Chez elle la langue parlée était l'arabe dialectal comme chez la famille Mokeddem. Le français écrit par Djebar n'est pas un français tout à fait normalisé. On dirait que, chez elle, la langue est un espace d'où résonnent des voix arabes et berbères, traduites en français par l'écrivaine. Elle se sert de mots archaïques et choisit souvent son vocabulaire dans un registre élevé.

Dans son autobiographie atypique L'amour la fantasia, publié en 1985, elle évoque non seulement sa jeunesse, mais surtout la Conquête de 1830 par les Français, qui est bien plutôt une défaite aux yeux des Algériens, et la guerre de L'indépendance nationale, la guerre d'Algérie. De cette façon, elle démontre d'une façon magistrale comment sa mémoire individuelle est liée intimement à la mémoire collective algérienne.

L'oeuvre de Djebar a été presque intégralement traduite en néerlandais, mais son autobiographie n'a jamais vu le jour dans la langue de Vondel. Il se peut que cela tienne à cette imbrication entre contenu et langue et aux références multiples à l'histoire coloniale. J'ai traduit moi-même un extrait de l'autobiographie que je considère comme un texte magnifique, un texte qui reflète le thème 
des relations de pouvoir entre l'arabe dialectal et le français et qui évoque le sentiment ambigu de ce don de la langue française. Le fragment s'intitule "La tunique de Nessus "7.

Le terme " tunique de Nessus " est utilisé pour désigner un cadeau empoisonné. Le TLF dit aussi qu'en référence à la légende d'Hercule, Tunique de Nessus signifie " Chose qui étreint ou qui ronge à l'intérieur. " Ces deux définitions peuvent s'appliquer à la langue française ou au rapport à la langue française chez Djebar. Elle utilise le mythe de la tunique qui brûle la peau de celui qui la met, pour évoquer cette langue de l'autre qui lui est en fait une “langue marâtre " (298), la " langue adverse " (300), la langue de "l'adversaire d'hier" (300). Le français est une langue qui colle à sa peau et qui fait mal à cause des champs de bataille d'autrefois, des exactions commises par le colonisateur aussi bien lors de la colonisation (les enfumades ${ }^{8}$ ) que lors de la guerre de décolonisation (la torture). Le français est " la langue encore coagulée des Autres " (302) qui a enveloppé l'écrivain dès l'enfance.

Le fragment " La tunique de Nessus" commence ainsi :

Le père, silhouette droite et le fez sur la tête, marche dans la rue du village ; sa main me tire et moi qui longtemps me croyait si fière - moi, la première de la famille à laquelle on achetait des poupées françaises, moi qui, devant le voile-suaire n'avais nul besoin de trépigner ou de baisser l'échine comme telle ou telle cousine, moi qui suprême coquetterie, en me voilant lors d'une noce d'été, m'imaginais me déguiser, puisque, définitivement, j'avais échappé à l'enfermement - je marche, fillette, au-dehors, main dans la main du père. (p. 297)

La phrase très longue se caractérise par un choix de mots qui saute aux yeux comme " baisser l'échine", mais aussi une image culturelle compliquée : "voile-suaire". Une image paradoxale qui relie le voile à la mort. La fillette échappe à l'enfermement, n'est 
pas obligée de porter le voile et va à l'école française ; c'est le père instituteur qui donne ce cadeau à sa fille, mais Djebar dira qu'il s'agit d'un cadeau empoisonné, étant donné que le français a tué et opprimé ses ancêtres. A l'inverse le français est aussi une langue libératrice et émancipatrice.

Comme chez Mokeddem, je veux m'arrêter aux moments textuels où la langue est porteuse de l'étrangeté à traduire. Là où le français porte la mémoire de la colonisation et des rapports de force entre colonisateur et colonisés, où Djebar, tout comme Mokeddem, évoque un territoire intermédiaire dans le texte. Chez Mokeddem c'est l'isthme, un lieu géographique, chez l'historienne Djebar il s'agit de mots qui évoquent l'histoire coloniale. Des mots qui réfèrent à un territoire où le colonisateur et le colonisé se combattent. Je dirais que ce combat se poursuit à l'intérieur de l'écrivain. Le rapport à la langue française chez Djebar est ambivalent, l'écrivain évoque une lutte mortelle entre l'arabe dialectal et le français, langue imposée par le colonisateur et par le père assimilé à la culture française.

Les mots qui véhiculent l'histoire coloniale sont : " préside ", " rebato" et " écharnement". Il s'agit de deux mots peu communs en français, à savoir " préside ", et " écharnement " et d'un mot emprunté à l'espagnol, choisis par l'auteur pour parler de son rapport à la langue française et à l'arabe dialectal. Le mot “ écharnement" a été choisi pour parler de la guerre de décolonisation.

Djebar explique son rapport à la langue française en écrivant :

Bien avant le débarquement français de 1830, durant des siècles autour des présides espagnols (Oran, Bougie, comme Tanger ou Ceuta, au Maroc) la guerre entre indigènes résistants et occupants souvent bloqués se faisait selon la tactique du " rebato" : point isolé d'où l'on attaquait, où l'on se repliait avant que, dans les trêves intermédiaires, le lieu devienne zone de cultures, ou de ravitaillement. (p. 299) 
Un préside est une enclave, une forteresse, construite au $19^{\mathrm{e}}$ siècle par les Espagnols colonisateurs. Il s'agit d'un territoire colonial protégé par une armée, servant souvent comme colonie pénitentiaire. La narratrice du fragment raconte que la population indigène au Maroc ou en Algérie, résistait par une tactique qui consistait à continuer à attaquer et à résister : la tactique de " Rebato ". Pendant les trêves, le territoire servait de zone de cultures.

Dans le fragment suivant la narratrice, qui rappelons-nous, écrit son autobiographie, compare la langue française, sa langue d'écriture à un " orgueilleux préside ", donc à une forteresse. La langue du colonisateur qui se bat avec les indigènes, la langue arabe qui se défend et qui attaque pour survivre. Aussi bien chez Djebar que chez Mokeddem, le rapport à la langue découle donc de la colonisation, leur langue constitue une mémoire littéraire de l'histoire coloniale.

\begin{abstract}
Après plus d'un siècle d'occupation française - qui finit, il y a peu, par un écharnement -, un territoire de langue subsiste entre deux peuples, entre deux mémoires ; la langue française, corps et voix, s'installe en moi comme un orgueilleux préside, tandis que la langue maternelle, toute en oralité, en hardes dépenaillées, résiste et attaque, entre deux essoufflements. (p. 299)
\end{abstract}

Ce qui est intéressant dans l'image de la tactique du " rebato ", c'est que le vainqueur change. Parfois c'est le colonisateur, parfois c'est l'indigène. De plus, l'indigène ne se laisse pas faire, il résiste et attaque lui aussi. Le dialecte arabe, la langue orale, est en haillons. C'est une langue non littéraire et non écrite. Pour écrire, Djebar ne possède que la langue de l'ancien colonisateur, qui installait des forteresses le long de la côte pour dominer les " indigènes ". Le français s'est installé comme une forteresse, un préside, mot délibérément choisi pour évoquer la colonisation. Pour évoquer son rapport au français Djebar a choisi donc l'image de la tu- 
nique de Nessus qui lui colle à la peau, et qui ronge de l'intérieur et l'image de la place forte, de la forteresse, qui est attaquée par l'arabe dialectal. Son for intérieur est donc comme une arène de combat entre différentes langues où aucune langue ne prend le dessus.

"Préside " et " rebato " ne posent pas de problèmes de traduction ; j'ai délibérément choisi le mot " presidio " en néerlandais etnon pas " fort " étant donné que le mot " presidio " porte aussi les échos de la colonisation.

Les différentes couches du mot " écharnement " du fragment cité posent par contre des problèmes de traduction . Djebar évoque la situation après le départ des Français, à l'indépendance de l'Algérie. L'occupation française finit, écrit-elle, par un " écharnement", et c'est ce mot étrange qui est intéressant. Echarnement signifie : "Action d'écharner les peaux, d'en ôter les chairs ; résultat de cette action. " (TLF). Le verbe écharner renvoie à une apparence dépouillée, à l'enlèvement de chair avec un couteau. Le terme réfère entre autres aux tanneurs. Mais quelle est la signification du mot dans le contexte du fragment choisi : " qui finit, il y a peu, par un écharnement" ? S'agit-il de la guerre d'Algérie ? D'une unité Algérie/France qui a été " écharnée " après la fin de la guerre en 1962 ? Est-ce la France qui est dépouillée, ou l'Algérie, ou les deux ? Qui mène l'action ? Est-ce que le couteau avec lequel on écharne, renvoie à la guerre d'Algérie? J'ai pensé à tout cela quand il me fallait une traduction pour ce mot " écharnement ". Il faudrait trouver un mot aussi rare en néerlandais, comme le mot (forgé) “ ontvlezing ". L'image fait mal, elle est très caractéristique des moyens d'expression de Djebar. Mais il apparaît clairement que la traduction inséparable de l'interprétation de l'implicite. A chaque fois il s'agit d'un choix du traducteur et de sa compréhension du texte. 


\section{Conclusion}

J'espère avoir montré comment " l'étranger " selon Ricoeur opère dans quelques fragments de textes. Chez Mokeddem le rapport au français est plutôt ludique, elle s'en sert pour l'incruster, pour l'embellir, pour la parer avec une oralité venant du désert. Chez Djebar, le rapport au français est plutôt une lutte mortelle. Mokeddem veut déconstruire les oppositions binaires, mais Djebar les crée en décrivant un rapport hiérarchique entre colonisateur et " indigène ", entre le français et l'arabe dialectal. Le vainqueur est tantôt le colonisateur, tantôt le colonisé.

C'est en réfléchissant à la traduction de termes ou d'expressions complexes et polysémiques forgés par les auteurs elles-mêmes, que j'ai pu analyser le rapport au français d'Assia Djebar et de Malika Mokeddem à travers les métaphores qu'elles donnent elles-mêmes de ce rapport. Pour un traducteur l'analyse d'un tel rapport est toujours indispensable afin de pourvoir rendre le texte dans toutes ses nuances et étrangetés.

\section{Notes}

1. Dora Sales Salvador, "Documentation as Ethics in Postcolonial Translation" in Translation Journal http://accurapid.com/journal/35documentation.htm, consulté en novembre 2006.

2. Cf. Susan Bassnett et Harish Trivedi (éd.), Postcolonial Translation. Theory and Practice, New York: Routledge 1999; Raoul J. Granqvist (éd.), Writing Back in/and Translation, Frankfurt am Main: Peter Lang 2006; Aline Remael et Ilse Logie (éd.) Translation as Creation: the Postcolonial Influence. Linguistica Antverpiensia 2/2003. 
3. Paul Ricoeur, "Défi et bonheur de la traduction", in Sur la traduction, Paris : Bayard 2004 ; traduction néerlandaise " De vertaling als uitdaging en als geluksgevoel " in Filter, tijdschrift over vertalen jaargang 15 nummer 1 mars 2008, traduit du français par Désirée Schyns.

4. Yolande Aline Helm (éd), Malika Mokeddem envers et contre tout, Paris : L'Harmattan 2000.

5. Malika Mokeddem, N'zid, Paris: Editions du Seuil 2001, traduction néerlandaise : Een vrouwelijke Odysseus, N'zid traduit du français par Désirée Schyns, Breda : De Geus, 2003. Le titre en néerlandais montre que chaque traduction constitue une négociation avec un éditeur. Etant donné que l'éditeur ne voulait pas publier la traduction avec le seul mot arabe " N'zid " comme titre, la traductrice a dû trouver un compromis. De cette façon le mot arabe si important dans le roman a été sauvegardé, mais en fin du titre néerlandais.

6. Cf. Rabia Redouane, "N'zid de Malika Mokeddem ou la (re)-naissance d'un être féminin " in International Journal of Francophone Studies volume 5, Issue 1, Avril 2002, p. 13-21.

7. Assia Djebar, "La tunique de Nessus" in L'amour la fantasia, Paris : Editions Albin Michel 1995 [Lattès 1985], p.297-302.

8. Dans différentes section de L'amour la fantasia et surtout dans " Femmes, enfants, bœufs couchés dans les grottes" (94-159), Djebar raconte comment les Français ont brisé les tribus résistantes rassemblées sous Abdelkader en les enfumant vifs dans des grottes. Cf ; aussi, Olivier Le Cour Grandmaison, Coloniser Exterminer. Sur la guerre et l'Etat colonial, Paris : Fayard 2005. 


\section{Bibliographie}

Susan Bassnett et Harish Trivedi (éd.), Postcolonial Translation. Theory and Practice, New York: Routledge, 1999.

Assia Djebar, "La tunique de Nessus" in L'amour la fantasia, Paris : Editions Albin Michel, 1995 [Lattès 1985], pp.297-302, traduction néerlandaise " De Nessusmantel " traduit du français par Désirée Schyns (traduction non publiée).

Raoul J. Granqvist (éd.), Writing Back in/and Translation, Frankfurt am Main: Peter Lang, 2006.

Yolande Aline Helm (éd), Malika Mokeddem envers et contre tout, Paris : L'Harmattan, 2000.

Olivier Le Cour Grandmaison, Coloniser Exterminer. Sur la guerre et l'Etat colonial, Paris : Fayard, 2005.

Malika Mokeddem, N'zid, Paris: Editions du Seuil 2001, traduction néerlandaise : Een vrouwelijke Odysseus, N'zid traduit du français par Désirée Schyns, Breda : De Geus, 2003.

Rabia Redouane, "N'zid de Malika Mokeddem ou la (re)-naissance d'un être féminin " in International Journal of Francophone Studies volume 5, Issue 1, Avril 2002, p. 13-21.

Aline Remael et Ilse Logie (éd.) Translation as Creation: the Postcolonial Influence. Linguistica Antverpiensia 2/2003.

Paul Ricoeur, "Défi et bonheur de la traduction", in Sur la traduction, Paris : Bayard, 2004 ; p. 7-20, traduction néerlandaise " De vertaling als uitdaging en als geluksgevoel " in Filter, tijdschrift over vertalen jaargang 15 nummer 1 mars 2008, traduit du français par Désirée Schyns, p. 71-75. 
Dora Sales Salvador, "Documentation as Ethics in Postcolonial Translation" in Translation Journal http://accurapid.com/journal/35documentation.htm, consulté en novembre 2006.

Désirée Schyns, Une écharde dans la gorge. L'évolution de la mémoire littéraire de la guerre d'Algérie (1954-1962) dans la fiction algérienne francophone (19582003), thèse soutenue à l'Université d'Amsterdam le 12 octobre 2007 (à paraître chez L'Harmattan à Paris).

Désirée Schyns, “ De Franse taal als oorlogsbuit. Een vertaald fragment uit N'zid van Malika Mokeddem" in Hilde Van Belle et Henri Bloemen (éd.), Vertaal! Over de didactiek van het literair vertalen, Leuven/Voorburg: Acco, 2004, p. 21-32. 